\title{
Application of Coupled Fixed Point Technique in Solving Integral Equations on Modified Intuitionistic Fuzzy Metric Spaces
}

\author{
Bhavana Deshpande and Amrish Handa \\ Department of Mathematics, Govt. P.G. Arts and Science College, Ratlam 457001, India \\ Correspondence should be addressed to Bhavana Deshpande; bhavnadeshpande@yahoo.com
}

Received 7 January 2014; Accepted 18 May 2014; Published 22 June 2014

Academic Editor: Rustom M. Mamlook

Copyright (C) 2014 B. Deshpande and A. Handa. This is an open access article distributed under the Creative Commons Attribution License, which permits unrestricted use, distribution, and reproduction in any medium, provided the original work is properly cited.

We establish a common coupled fixed point theorem for weakly compatible mappings on modified intuitionistic fuzzy metric spaces. As an application of our result, we study the existence and uniqueness of the solution to a nonlinear Fredholm integral equation. We also give an example to demonstrate our result.

\section{Introduction}

The concept of fuzzy metric space has been introduced in several ways. In [1], Kramosil and Michalek introduced the concept of fuzzy metric space. Later on, it is modified by George and Veeramani [2] with the help of continuous $t$ norms and they defined the Hausdorff topology of fuzzy metric spaces.

Atanassov [3] introduced and studied the concept of intuitionistic fuzzy sets as a generalization of fuzzy sets. Alaca et al. [4] using the idea of intuitionistic fuzzy sets defined the notion of intuitionistic fuzzy metric space with the help of continuous $t$-norms and continuous $t$-conorms as a generalization of fuzzy metric space due to Kramosil and Michalek [1]. In [5], Park generalized the notion of fuzzy metric space given by George and Veeramani [2] and introduced the notion of intuitionistic fuzzy metric space.

Gregori et al. [6] pointed out that topologies generated by fuzzy metric and intuitionistic fuzzy metric coincide. In view of this observation, Saadati et al. [7] modified the notion of intuitionistic fuzzy metric and defined the notion of modified intuitionistic fuzzy metric spaces with the help of continuous $t$-representable.

Bhaskar and Lakshmikantham [8] introduced the notion of coupled fixed point and mixed monotone mappings and gave some coupled fixed point theorems. As an application, they study the existence and uniqueness of solution for periodic boundary value problems. Lakshmikantham and Ciric [9] introduced the concept of coupled coincidence point and proved some common coupled fixed point theorems. Sedghi et al. [10] gave a coupled fixed point theorem for contractions in fuzzy metric space, which was further generalized by $\mathrm{Hu}$ [11]. In [12], Hu et al. improved, rectified, and generalized the result obtained in [11].

On the other hand, many scientific and engineering problems can be described by integral equations. Initial and boundary value problems can be transformed into Volterra or Fredholm integral equations. Integral equations can also be created by many mathematical physics models such as diffraction problems, scattering in quantum mechanics, conformal mapping, and water wave. Integral equations or integro-differential equations can be applied in science and engineering. Many areas that are described by integral equations are Volterra's population growth model, biological species living together, propagation of stocked fish in a new lake, the heat radiation, and so forth.

Very recently, Deshpande et al. [13] proved a common fixed point theorem for mappings under $\phi$-contractive conditions on intuitionistic fuzzy metric spaces. As an application, 
they study the existence and uniqueness of the solution to a nonlinear Fredholm integral equation.

In this paper, we prove a common coupled fixed point theorem for weakly compatible mappings on modified intuitionistic fuzzy metric spaces. As an application of our result, we study the existence and uniqueness of the solution to a nonlinear Fredholm integral equation, which arise naturally in the theory of signal processing, linear forward modeling, and inverse problems. We also give an example to validate our result. We extend and generalize the results of $\mathrm{Hu}$ [11], Hu et al. [12], and Sedghi et al. [10] in the settings of modified intuitionistic fuzzy metric spaces. The result is the genuine generalization of the result of Deshpande et al. [13].

\section{Preliminaries}

Lemma 1 (Deschrijver and Kerre [14]). Consider the set $L^{*}$ and operation $\leq_{L^{*}}$ defined by

$$
L^{*}=\left\{\left(x_{1}, x_{2}\right):\left(x_{1}, x_{2}\right) \in[0,1]^{2}, x_{1}+x_{2} \leq 1\right\},
$$

$\left(x_{1}, x_{2}\right) \leq_{L^{*}}\left(y_{1}, y_{2}\right) \Leftrightarrow x_{1} \leq y_{1}$, and $x_{2} \geq y_{2}$ for every $\left(x_{1}, x_{2}\right),\left(y_{1}, y_{2}\right) \in L^{*}$. Then $\left(L^{*}, \leq_{L^{*}}\right)$ is a complete lattice.

Definition 2 (Atanassov [3]). An intuitionistic fuzzy set $\mathscr{A}_{\zeta, \eta}$ in a universe $U$ is an object $\mathscr{A}_{\zeta, \eta}=\left\{\zeta_{\mathscr{A}}(u), \eta_{\mathscr{A}}(u)\right\}$, where, for all $u \in U, \zeta_{\mathscr{A}}(u) \in[0,1]$ and $\eta_{\mathscr{A}}(u) \in[0,1]$ are called the membership degree and nonmembership degree, respectively, of $u$ in $\mathscr{A}_{\zeta, \eta}$ and further they satisfy $\zeta_{\mathscr{A}}(u)+$ $\eta_{\mathscr{A}}(u) \leq 1$. For every $z_{i}=\left(x_{i}, y_{i}\right) \in L^{*}$, if $c_{i} \in[0,1]$ such that $\sum_{j=1}^{n} c_{j}=1$, then it is easy to see that

$$
\begin{aligned}
& c_{1}\left(x_{1}, y_{1}\right)+\cdots+c_{n}\left(x_{n}, y_{n}\right) \\
& =\sum_{j=1}^{n} c_{j}\left(x_{j}, y_{j}\right)=\left(\sum_{j=1}^{n} c_{j} x_{j}, \sum_{j=1}^{n} c_{j} y_{j}\right) \in L^{*} .
\end{aligned}
$$

We denote its units by $0_{L^{*}}=(0,1)$ and $1_{L^{*}}=(1,0)$. Classically, a triangular norm $*=T$ on $[0,1]$ is defined as an increasing, commutative, associative mapping $T:[0,1]^{2} \rightarrow$ $[0,1]$ satisfying $T(1, x)=1 * x=x$, for all $x \in[0,1]$. A triangular conorm $S=\diamond$ is defined as an increasing, commutative, associative mapping $S:[0,1]^{2} \rightarrow[0,1]$ satisfying $S(0, x)=0 \diamond x=x$, for all $x \in[0,1]$. Using the lattice $\left(L^{*}, \leq_{L^{*}}\right)$, these definitions can be straightforwardly extended.

Definition 3 (Deschrijver et al. [15]). A triangular norm ( $t$ norm) on $L^{*}$ is a mapping $\mathscr{T}:\left(L^{*}\right)^{2} \rightarrow L^{*}$ satisfying the following conditions:

$\left(\forall x \in L^{*}\right)\left(\mathscr{T}\left(x, 1_{L^{*}}\right)=x\right)$ (boundary condition), $\left(\forall(x, y) \in\left(L^{*}\right)^{2}\right)(\mathscr{T}(x, y)=\mathscr{T}(y, x))$ (commutativity),

$\left(\forall(x, y, z) \in\left(L^{*}\right)^{3}\right)(\mathscr{T}(x, \mathscr{T}(y, z))=\mathscr{T}(\mathscr{T}(x, y), z))$ (associativity),

$\left(\forall\left(x, x^{\prime}, y, y^{\prime}\right) \in\left(L^{*}\right)^{4}\right)\left(x \leq_{L^{*}} x^{\prime}\right.$ and $y \leq_{L^{*}} y^{\prime} \Rightarrow$ $\left.\mathscr{T}(x, y) \leq_{L^{*}} \mathscr{T}\left(x^{\prime}, y^{\prime}\right)\right)$ (monotonicity).
Definition 4 (Deschrijver and Kerre [14] and Deschrijver et al. [15]). A continuous $t$-norm $\mathscr{T}$ on $L^{*}$ is called continuous $t$-representable if and only if there exist a continuous $t$-norm $*$ and a continuous $t$-conorm $\diamond$ on $[0,1]$ such that, for all $x=\left(x_{1}, x_{2}\right), y=\left(y_{1}, y_{2}\right) \in L^{*}$,

$$
\mathscr{T}(x, y)=\left(x_{1} * y_{1}, x_{2} \diamond y_{2}\right) .
$$

Now define a sequence $\mathscr{T}^{n}$ recursively by $\mathscr{T}^{1}=\mathscr{T}$ and

$$
\mathscr{T}^{n}\left(x^{(1)}, \ldots, x^{(n+1)}\right)=\mathscr{T}\left(\mathscr{T}^{n-1}\left(x^{(1)}, \ldots, x^{(n)}\right), x^{(n+1)}\right),
$$

for $n \geq 2$ and $x^{(i)} \in L^{*}$.

Definition 5 (Deschrijver and Kerre [14] and Deschrijver et al. [15]). A negator on $L^{*}$ is any decreasing mapping $\mathcal{N}: L^{*} \rightarrow$ $L^{*}$ satisfying $\mathcal{N}\left(0_{L^{*}}\right)=1_{L^{*}}$ and $\mathcal{N}\left(1_{L^{*}}\right)=0_{L^{*}}$. If $\mathcal{N}(\mathcal{N}(x))=$ $x$, for all $x \in L^{*}$, then $\mathcal{N}$ is called an involutive negator. A negator on $[0,1]$ is a decreasing mapping $N:[0,1] \rightarrow[0,1]$ satisfying $N(0)=1$ and $N(1)=0 . N_{s}$ denotes the standard negator on $[0,1]$ defined as, for all $x \in[0,1], N_{s}(x)=1-x$.

Definition 6 (Saadati et al. [7]). Let $M, N$ be fuzzy sets from $X^{2} \times(0,+\infty)$ to $[0,1]$ such that $M(x, y, t)+N(x, y, t) \leq 1$ for all $x, y \in X$ and $t>0$. The 3 -tuple $\left(X, \mathscr{M}_{M, N}, \mathscr{T}\right)$ is said to be a modified intuitionistic fuzzy metric space if $X$ is an arbitrary (nonempty) set, $\mathscr{T}$ is a continuous $t$-representable, and $\mathscr{M}_{M, N}$ is a mapping $X^{2} \times(0,+\infty) \rightarrow L^{*}$ satisfying the following conditions for every $x, y, z \in X$ and $t, s>0$ :

(a) $\mathscr{M}_{M, N}(x, y, t)>_{L^{*}} 0_{L^{*}}$,

(b) $\mathscr{M}_{M, N}(x, y, t)=1_{L^{*}}$ if and only if $x=y$,

(c) $\mathscr{M}_{M, N}(x, y, t)=\mathscr{M}_{M, N}(y, x, t)$,

(d) $\mathscr{M}_{M, N}(x, y, t+s) \geq_{L^{*}} \mathscr{T}\left(\mathscr{M}_{M, N}(x, z, t), \mathscr{M}_{M, N}(z, y, s)\right)$,

(e) $\mathscr{M}_{M, N}(x, y, \cdot):(0, \infty) \rightarrow L^{*}$ is continuous.

In this case, $\mathscr{M}_{M, N}$ is called a modified intuitionistic fuzzy metric. Here,

$$
\mathscr{M}_{M, N}(x, y, t)=(M(x, y, t), N(x, y, t)) .
$$

Example 7 (Saadati et al. [7]). Let $(X, d)$ be a metric space. Denote $\mathscr{T}(a, b)=\left(a_{1} b_{1}, \min \left\{a_{2}+b_{2}, 1\right\}\right)$ for all $a=\left(a_{1}, a_{2}\right)$ and $b=\left(b_{1}, b_{2}\right) \in L^{*}$ and let $M$ and $N$ be fuzzy sets on $X^{2} \times(0, \infty)$ defined as follows:

$$
\begin{aligned}
\mathscr{M}_{M, N}(x, y, t) & =(M(x, y, t), N(x, y, t)) \\
& =\left(\frac{h t^{n}}{h t^{n}+m d(x, y)}, \frac{m d(x, y)}{h t^{n}+m d(x, y)}\right),
\end{aligned}
$$

for all $t, h, m, n \in R^{+}$. Then $\left(X, \mathscr{M}_{M, N}, \mathscr{T}\right)$ is a modified intuitionistic fuzzy metric space.

Example 8 (Saadati et al. [7]). Let $X=N$. Denote $\mathscr{T}(a, b)=$ $\left(\max \left\{0, a_{1}+b_{1}-1\right\}, a_{2}+b_{2}-a_{2} b_{2}\right)$ for all $a=\left(a_{1}, a_{2}\right)$ and 
$b=\left(b_{1}, b_{2}\right) \in L^{*}$ and let $M$ and $N$ be fuzzy sets on $X^{2} \times(0, \infty)$ defined as follows:

$$
\begin{aligned}
\mathscr{M}_{M, N}(x, y, t) & =(M(x, y, t), N(x, y, t)) \\
& = \begin{cases}\left(\frac{x}{y}, \frac{y-x}{y}\right) & \text { if } x \leq y, \\
\left(\frac{y}{x}, \frac{x-y}{x}\right) & \text { if } y \leq x,\end{cases}
\end{aligned}
$$

for all $x, y \in X$ and $t>0$. Then $\left(X, \mathscr{M}_{M, N}, \mathscr{T}\right)$ is a modified intuitionistic fuzzy metric space.

Lemma 9 (Fang [16]). We say that the intuitionistic fuzzy metric space $\left(X, \mathscr{M}_{M, N}, \mathscr{T}\right)$ has property $(C)$, if it satisfies the following condition: $\mathscr{M}_{M, N}(x, y, t)=C$ for all $t>0$ implies $C=1_{L^{*}}$.

Remark 10. Throughout this paper, $\left(X, \mathscr{M}_{M, N}, \mathscr{T}\right)$ is a modified intuitionistic fuzzy metric space with property $(C)$.

Definition 11 (Saadati et al. [7]). A sequence $\left\{x_{n}\right\}$ in a modified intuitionistic fuzzy metric space $\left(X, \mathscr{M}_{M, N}, \mathscr{T}\right)$ is called a Cauchy sequence if, for each $0<\varepsilon<1$ and $t>0$, there exists $n_{0} \in N$ such that

$$
\mathscr{M}_{M, N}\left(x_{n}, x_{m}, t\right)>_{L^{*}}\left(N_{s}(\varepsilon), \varepsilon\right),
$$

and, for each $n, m \geq n_{0}$, here $N_{s}$ is the standard negator. The sequence $\left\{x_{n}\right\}$ is said to be convergent to $x \in X$ in the modified intuitionistic fuzzy metric space $\left(X, \mathscr{M}_{M, N}, \mathscr{T}\right)$ and denoted by $x_{n} \stackrel{\mathscr{M}_{M, N}}{\longrightarrow} x$, if $\mathscr{M}_{M, N}\left(x_{n}, x, t\right) \rightarrow 1_{L^{*}}$ whenever $n \rightarrow \infty$ for every $t>0$. A modified intuitionistic fuzzy metric space is said to be complete if and only if every Cauchy sequence is convergent.

Lemma 12 (Saadati and Park [17]). Let $\mathscr{M}_{M, N}$ be a modified intuitionistic fuzzy metric. Then, for any $t>0, \mathscr{M}_{M, N}(x, y, t)$ is nondecreasing with respect to $t$, in $\left(L^{*}, \leq_{L^{*}}\right)$ for all $x, y$ in $X$.

Definition 13 (Saadati et al. [7]). Let $\left(X, \mathscr{M}_{M, N}, \mathscr{T}\right)$ be a modified intuitionistic fuzzy metric space. For $t>0$, define the open ball $B(x, r, t)$ with center $x \in X$ and radius $0<r<1$, as

$$
B(x, r, t)=\left\{y \in X: \mathscr{M}_{M, N}(x, y, t)>_{L^{*}}\left(N_{s}(r), r\right)\right\} .
$$

A subset $A \subset X$ is called open if, for each $x \in A$, there exist $t>0$ and $0<r<1$ such that $B(x, r, t) \subset A$. Let $\tau_{M, N}$ denote the family of all open subsets of $X . \tau_{M, N}$ is called the topology induced by modified intuitionistic fuzzy metric. This topology is Hausdorff.

Definition 14 (Saadati et al. [7]). Let $\left(X, \mathscr{M}_{M, N}, \mathscr{T}\right)$ be a modified intuitionistic fuzzy metric space. $\mathscr{M}$ is said to be continuous on $X \times X \times(0, \infty)$ if

$$
\lim _{n \rightarrow \infty} \mathscr{M}_{M, N}\left(x_{n}, y_{n}, t_{n}\right)=\mathscr{M}_{M, N}(x, y, t),
$$

whenever a sequence $\left\{\left(x_{n}, y_{n}, t_{n}\right)\right\}$ in $X \times X \times(0, \infty)$ converges to a point $(x, y, t) \in X \times X \times(0, \infty)$, that is;

$$
\begin{aligned}
& \lim _{n \rightarrow \infty} \mathscr{M}_{M, N}\left(x_{n}, y, t\right)=\mathscr{M}_{M, N}(x, y, t), \\
& \lim _{n \rightarrow \infty} \mathscr{M}_{M, N}\left(x, y_{n}, t\right)=\mathscr{M}_{M, N}(x, y, t), \\
& \lim _{n \rightarrow \infty} \mathscr{M}_{M, N}\left(x, y, t_{n}\right)=\mathscr{M}_{M, N}(x, y, t) .
\end{aligned}
$$

Lemma 15 (Saadati et al. [7]). Let $\left(X, \mathscr{M}_{M, N}, \mathscr{T}\right)$ be a modified intuitionistic fuzzy metric space. Then $M$ is continuous function on $X \times X \times(0, \infty)$.

Definition 16 (Bhaskar and Lakshmikantham [8]). An element $(x, y) \in X \times X$ is called a coupled fixed point of the mapping $F: X \times X \rightarrow X$ if

$$
F(x, y)=x, \quad F(y, x)=y .
$$

Definition 17 (Lakshmikantham and Ciric [9]). Let $X$ be a nonempty set. An element $(x, y) \in X \times X$ is called a coupled coincidence point of the mappings $F: X \times X \rightarrow X$ and $g: X \rightarrow X$ if

$$
F(x, y)=g(x), \quad F(y, x)=g(y) .
$$

Definition 18 (Lakshmikantham and Ciric [9]). Let $X$ be a nonempty set. An element $(x, y) \in X \times X$ is called a common coupled fixed point of the mappings $F: X \times X \rightarrow X$ and $g: X \rightarrow X$ if

$$
x=F(x, y)=g(x), \quad y=F(y, x)=g(y) .
$$

Definition 19 (Lakshmikantham and Ciric [9]). Let $X$ be a nonempty set. An element $x \in X$ is called a common fixed point of the mappings $F: X \times X \rightarrow X$ and $g: X \rightarrow X$ if

$$
x=F(x, x)=g(x) .
$$

Definition 20 (Lakshmikantham and Ciric [9]). Let $X$ be a nonempty set. The mappings $F: X \times X \rightarrow X$ and $g: X \rightarrow X$ are said to be commutative if

$$
g F(x, y)=F(g x, g y), \quad \forall(x, y) \in X \times X .
$$

Definition 21 (Fang [18]). Let $\left(X, \mathscr{M}_{M, N}, \mathscr{T}\right)$ be a modified intuitionistic fuzzy metric space. The mappings $F: X \times X \rightarrow$ $X$ and $g: X \rightarrow X$ are said to be compatible if

$$
\begin{aligned}
& \lim _{n \rightarrow \infty} \mathscr{M}_{M, N}\left(g F\left(x_{n}, y_{n}\right), F\left(g x_{n}, g y_{n}\right), t\right)=1_{L^{*}}, \\
& \lim _{n \rightarrow \infty} \mathscr{M}_{M, N}\left(g F\left(y_{n}, x_{n}\right), F\left(g y_{n}, g x_{n}\right), t\right)=1_{L^{*}},
\end{aligned}
$$

for all $t>0$, whenever $\left\{x_{n}\right\}$ and $\left\{y_{n}\right\}$ are sequences in $X$ such that

$$
\begin{gathered}
\lim _{n \rightarrow \infty} F\left(x_{n}, y_{n}\right)=\lim _{n \rightarrow \infty} g\left(x_{n}\right)=x, \\
\lim _{n \rightarrow \infty} F\left(y_{n}, x_{n}\right)=\lim _{n \rightarrow \infty} g\left(y_{n}\right)=y, \quad \forall x, y \in X .
\end{gathered}
$$

Definition 22 (Abbas et al. [19]). Let $X$ be a nonempty set. The mappings $F: X \times X \rightarrow X$ and $g: X \rightarrow X$ are called weakly compatible mappings if $F(x, y)=g(x), F(y, x)=$ $g(y)$ implies that $g F(x, y)=F(g x, g y)$ and $g F(y, x)=$ $F(g y, g x)$, for all $x, y \in X$. 


\section{Main Results}

Definition 23. Let $\sup _{0<t<1} \mathscr{T}(t, t)=1_{L^{*}}$. A continuous $t$ representable $\mathscr{T}$ is said to be continuous $t$-representable of $\mathrm{H}$ type if the family of functions $\left\{\mathscr{T}^{m}(t)\right\}_{m=1}^{\infty}$ is equicontinuous at $t=1_{L^{*}}$, where

$$
\begin{array}{r}
\mathscr{T}^{1}(t)=\mathscr{T}(t, t), \quad \mathscr{T}^{m+1}(t)=\mathscr{T}\left(t, \mathscr{T}^{m}(t)\right), \\
m=1,2, \ldots, t \in L^{*} .
\end{array}
$$

Obviously, $\mathscr{T}$ is a H-type $t$-representable if and only if, for any $0<\lambda<1$, there exists $0<\mu<1$ such that

$$
\mathscr{T}^{m}(t)>_{L^{*}}\left(N_{s}(\lambda), \lambda\right), \quad \forall m \in N \text {, when } t>_{L^{*}}\left(N_{s}(\mu), \mu\right) .
$$

Remark 24. In a modified intuitionistic fuzzy metric space $\left(X, \mathscr{M}_{M, N}, \mathscr{T}\right)$, whenever $\mathscr{M}_{M, N}(x, y, t)>_{L^{*}}\left(N_{s}(r), r\right)$ for $x, y \in X, t>0$ and $0<r<1$, we can find a $t_{0}, 0<t_{0}<t$, such that $\mathscr{M}_{M, N}\left(x, y, t_{0}\right)>\left(N_{s}(r), r\right)$.

Remark 25. For convenience, we denote

$$
\left[\mathscr{M}_{M, N}(x, y, t)\right]^{n}=\mathscr{T}^{n-1}\left(\mathscr{M}_{M, N}(x, y, t)\right),
$$

for all $n \in N$.

Definition 26. Let $\left(X, \mathscr{M}_{M, N}, \mathscr{T}\right)$ be a modified intuitionistic fuzzy metric space. $\mathscr{M}_{M, N}$ is said to satisfy the $p$-property on $X^{2} \times(0, \infty)$ if

$$
\lim _{p \rightarrow \infty}\left[\mathscr{M}_{M, N}\left(x, y, k^{p} t\right)\right]^{p}=1_{L^{*}}
$$

whenever $x, y \in X, k>1$, and $p \in N$.

Lemma 27. Let $\left(X, \mathscr{M}_{M, N}, \mathscr{T}\right)$ be a modified intuitionistic fuzzy metric space and let $\mathscr{M}_{M, N}$ satisfy the p-property; then

$$
\lim _{t \rightarrow \infty} \mathscr{M}_{M, N}(x, y, t)=1_{L^{*}}, \quad \forall x, y \in X .
$$

Proof. If not, since $\mathscr{M}_{M, N}(x, y, \cdot)$ is nondecreasing, there exists $x_{0}, y_{0} \in X$ such that

$$
\lim _{t \rightarrow \infty} \mathscr{M}_{M, N}\left(x_{0}, y_{0}, t\right)=\left(\lambda, N_{s}(\lambda)\right)<1_{L^{*}}
$$

and then, for $k>1, k^{p} t \rightarrow \infty$ when $p \rightarrow \infty$ as $t>0$ and we get

$$
\lim _{p \rightarrow \infty}\left[\mathscr{M}_{M, N}\left(x_{0}, y_{0}, k^{p} t\right)\right]^{p}=0_{L^{*}}
$$

which is a contradiction.

Remark 28. Condition (22) cannot guarantee the p-property. See the following example.

Example 29. Let $(X, d)$ be an ordinary metric space and let $\psi$ be defined as follows:

$$
\psi(t)= \begin{cases}\alpha \sqrt{t}, & 0 \leq t \leq 4, \\ 1-\frac{1}{\ln t}, & t>4,\end{cases}
$$

where $\alpha=(1 / 2)(1-(1 / \ln 4))$. Then $\psi(t)$ is continuous and increasing in $(0, \infty), \psi(t) \in(0,1)$ and $\lim _{t \rightarrow \infty} \psi(t)=1$. Let

$$
\mathscr{M}_{M, N}(x, y, t)=\left([\psi(t)]^{d(x, y)}, 1-[\psi(t)]^{d(x, y)}\right),
$$

for all $x, y \in X$ and $t>0$, and $\mathscr{T}(a, b)=\left(a_{1} b_{1}, a_{2}+b_{2}-a_{2} b_{2}\right)$, for all $a=\left(a_{1}, a_{2}\right)$ and $b=\left(b_{1}, b_{2}\right) \in L^{*}$. Then $\left(X, \mathscr{M}_{M, N}, \mathscr{T}\right)$ is a modified intuitionistic fuzzy metric space with

$$
\begin{aligned}
\lim _{t \rightarrow \infty} & \mathscr{M}_{M, N}(x, y, t) \\
= & \lim _{t \rightarrow \infty}\left([\psi(t)]^{d(x, y)}, 1-[\psi(t)]^{d(x, y)}\right) \\
& =1_{L^{*}}, \quad \forall x, y \in X .
\end{aligned}
$$

But, for any $x \neq y, k>1$, and $t>0$,

$$
\begin{aligned}
\lim _{p \rightarrow \infty}[ & \left.\mathscr{M}_{M, N}\left(x, y, k^{p} t\right)\right]^{p} \\
= & \left(\lim _{p \rightarrow \infty}\left[\psi\left(k^{p} t\right)\right]^{d(x, y) \cdot p},\right. \\
& \left.\lim _{p \rightarrow \infty}\left[1-\left[\psi\left(k^{p} t\right)\right]^{d(x, y) \cdot p}\right]\right) \\
= & \left(\lim _{p \rightarrow \infty}\left[1-\frac{1}{\ln \left(k^{p} t\right)}\right]^{d(x, y) \cdot p},\right. \\
= & \left(e_{p \rightarrow \infty}^{-d(x, y) / \ln k}, 1-e^{-d(x, y) / \ln k}\right) \\
\neq & \left.\left.1_{L^{*} .}\left[1-\frac{1}{\ln \left(k^{p} t\right)}\right]^{p \cdot d(x, y)}\right]\right)
\end{aligned}
$$

Define $\Phi=\left\{\phi: R^{+} \rightarrow R^{+}\right\}$, where $R^{+}=[0,+\infty)$, and each $\phi \in \Phi$ satisfies the following conditions:

$(\phi-1) \phi$ is nondecreasing,

$(\phi-2) \phi$ is continuous,

$(\phi-3) \sum_{n=0}^{\infty} \phi^{n}(t)<+\infty$ for all $t>0$, where $\phi^{n+1}(t)=$ $\phi^{n}(\phi(t)), n \in N$.

It is easy to prove that if $\phi \in \Phi$, then $\phi(t)<t$ for all $t>0$.

Lemma 30. Let $\left(X, \mathscr{M}_{M, N}, \mathscr{T}\right)$ be a modified intuitionistic fuzzy metric space, where $\mathscr{T}$ is a continuous t-representable of $H$-type. If there exists $\phi \in \Phi$, such that if

$$
\mathscr{M}_{M, N}(x, y, \phi(t)) \geq_{L^{*}} \mathscr{M}_{M, N}(x, y, t), \quad \forall t>0,
$$

then $x=y$.

Proof. Since $t>0$ and $t>\phi(t)$. Using the monotony of $\mathscr{M}_{M, N}$, we have $\mathscr{M}_{M, N}(x, y, t) \geq_{L^{*}} \mathscr{M}_{M, N}(x, y, \phi(t))$. Using (29) and the definition of modified intuitionistic fuzzy metric, we have $x=y$.

Theorem 31. Let $\left(X, \mathscr{M}_{M, N}, \mathscr{T}\right)$ be a modified intuitionistic fuzzy metric space, where $\mathscr{T}$ is a continuous t-representable of 
H-type satisfying (22). Let $F: X \times X \rightarrow X$ and $g: X \rightarrow X$ be two weakly compatible mappings and there exists $\phi \in \Phi$ such that

$$
\begin{aligned}
& \mathscr{M}_{M, N}(F(x, y), F(u, v), \phi(t)) \\
& \geq_{L^{*}} \mathscr{T}\left(\mathscr{M}_{M, N}(g x, g u, t), \mathscr{M}_{M, N}(g y, g v, t)\right),
\end{aligned}
$$

for all $x, y, u, v \in X$ and $t>0$. Suppose that $F(X \times X) \subseteq g(X)$ and $F(X \times X)$ or $g(X)$ is complete. Then there exists a unique $x \in X$ such that $x=g(x)=F(x, x)$.

Proof. Let $x_{0}, y_{0} \in X$ be two arbitrary points in $X$. Since $F(X \times X) \subseteq g(X)$, we can choose $x_{1}, y_{1} \in X$ such that

$$
g x_{1}=F\left(x_{0}, y_{0}\right), \quad g y_{1}=F\left(y_{0}, x_{0}\right) .
$$

Continuing in this way, we can construct two sequences $\left\{x_{n}\right\}$ and $\left\{y_{n}\right\}$ in $X$ such that

$$
g x_{n+1}=F\left(x_{n}, y_{n}\right), \quad g y_{n+1}=F\left(y_{n}, x_{n}\right), \quad \forall n \geq 0
$$

The proof is divided into 4 steps.

Step 1. Prove that $\left\{g x_{n}\right\}$ and $\left\{g y_{n}\right\}$ are Cauchy sequences.

Since $\mathscr{T}$ is a continuous $t$-representable of H-type, therefore, for any $\lambda>0$, there exists a $\mu>0$ such that

$$
\mathscr{T}^{m-1}\left(N_{s}(\mu), \mu\right) \geq_{L^{*}}\left(N_{s}(\lambda), \lambda\right), \quad \forall m \in N
$$

Since $\lim _{t \rightarrow \infty} \mathscr{M}_{M, N}(x, y, t)=1_{L^{*}}$ for all $x, y \in X$, there exists $t_{0}>0$ such that

$$
\begin{aligned}
& \mathscr{M}_{M, N}\left(g x_{0}, g x_{1}, t_{0}\right) \geq_{L^{*}}\left(N_{s}(\mu), \mu\right), \\
& \mathscr{M}_{M, N}\left(g y_{0}, g y_{1}, t_{0}\right) \geq_{L^{*}}\left(N_{s}(\mu), \mu\right) .
\end{aligned}
$$

On the other hand, since $\phi \in \Phi$, by condition $(\phi-3)$, we have $\sum_{n=1}^{\infty} \phi^{n}\left(t_{0}\right)<\infty$. Then, for any $t>0$, there exists $n_{0} \in N$ such that

$$
t>\sum_{k=n_{0}}^{\infty} \phi^{k}\left(t_{0}\right)
$$

From condition (30), we have

$$
\begin{aligned}
\mathscr{M}_{M, N} & \left(g x_{1}, g x_{2}, \phi\left(t_{0}\right)\right) \\
& =\mathscr{M}_{M, N}\left(F\left(x_{0}, y_{0}\right), F\left(x_{1}, y_{1}\right), \phi\left(t_{0}\right)\right) \\
& \geq_{L^{*}} \mathscr{T}\left(\mathscr{M}_{M, N}\left(g x_{0}, g x_{1}, t_{0}\right), \mathscr{M}_{M, N}\left(g y_{0}, g y_{1}, t_{0}\right)\right), \\
\mathscr{M}_{M, N} & \left(g y_{1}, g y_{2}, \phi\left(t_{0}\right)\right) \\
& =\mathscr{M}_{M, N}\left(F\left(y_{0}, x_{0}\right), F\left(y_{1}, x_{1}\right), \phi\left(t_{0}\right)\right) \\
& \geq_{L^{*}} \mathscr{T}\left(\mathscr{M}_{M, N}\left(g y_{0}, g y_{1}, t_{0}\right), \mathscr{M}_{M, N}\left(g x_{0}, g x_{1}, t_{0}\right)\right) .
\end{aligned}
$$

Similarly, we can also get

$$
\begin{gathered}
\mathscr{M}_{M, N}\left(g x_{2}, g x_{3}, \phi^{2}\left(t_{0}\right)\right) \\
=\mathscr{M}_{M, N}\left(F\left(x_{1}, y_{1}\right), F\left(x_{2}, y_{2}\right), \phi^{2}\left(t_{0}\right)\right) \\
\geq_{L^{*}} \mathscr{T}\left(\mathscr{M}_{M, N}\left(g x_{1}, g x_{2}, \phi\left(t_{0}\right)\right),\right. \\
\left.\mathscr{M}_{M, N}\left(g y_{1}, g y_{2}, \phi\left(t_{0}\right)\right)\right) \\
\geq_{L^{*}} \mathscr{T}\left(\left[\mathscr{M}_{M, N}\left(g x_{0}, g x_{1}, t_{0}\right)\right]^{2},\right. \\
\left.\quad\left[\mathscr{M}_{M, N}\left(g y_{0}, g y_{1}, t_{0}\right)\right]^{2}\right), \\
\mathscr{M}_{M, N}\left(g y_{2}, g y_{3}, \phi^{2}\left(t_{0}\right)\right) \\
=\mathscr{M}_{M, N}\left(F\left(y_{1}, x_{1}\right), F\left(y_{2}, x_{2}\right), \phi^{2}\left(t_{0}\right)\right) \\
\geq_{L^{*}} \mathscr{T}\left(\mathscr{M}_{M, N}\left(g y_{1}, g y_{2}, \phi\left(t_{0}\right)\right),\right. \\
\left.\mathscr{M}_{M, N}\left(g x_{1}, g x_{2}, \phi\left(t_{0}\right)\right)\right) \\
\geq_{L^{*}} \mathscr{T}\left(\left[\mathscr{M}_{M, N}\left(g y_{0}, g y_{1}, t_{0}\right)\right]^{2},\right. \\
\left.\left[\mathscr{M}_{M, N}\left(g x_{0}, g x_{1}, t_{0}\right)\right]^{2}\right) .
\end{gathered}
$$

Continuing in the same way, we can get

$$
\begin{gathered}
\mathscr{M}_{M, N}\left(g x_{n}, g x_{n+1}, \phi^{n}\left(t_{0}\right)\right) \\
\geq_{L^{*}} \mathscr{T}\left(\left[\mathscr{M}_{M, N}\left(g x_{0}, g x_{1}, t_{0}\right)\right]^{2^{n-1}},\right. \\
\left.\left[\mathscr{M}_{M, N}\left(g y_{0}, g y_{1}, t_{0}\right)\right]^{2^{n-1}}\right), \\
\mathscr{M}_{M, N}\left(g y_{n}, g y_{n+1}, \phi^{n}\left(t_{0}\right)\right) \\
\geq_{L^{*}} \mathscr{T}\left(\left[\mathscr{M}_{M, N}\left(g y_{0}, g y_{1}, t_{0}\right)\right]^{2^{n-1}},\right. \\
\left.\left[\mathscr{M}_{M, N}\left(g x_{0}, g x_{1}, t_{0}\right)\right]^{2^{n-1}}\right) .
\end{gathered}
$$

Now, from (33), (34), and (35), for $m>n \geq n_{0}$, we have

$$
\begin{gathered}
\mathscr{M}_{M, N}\left(g x_{n}, g x_{m}, t\right) \\
\geq_{L^{*}} \mathscr{M}_{M, N}\left(g x_{n}, g x_{m}, \sum_{k=n_{0}}^{\infty} \phi^{k}\left(t_{0}\right)\right) \\
\geq_{L^{*}} \mathscr{M}_{M, N}\left(g x_{n}, g x_{m}, \sum_{k=n}^{m-1} \phi^{k}\left(t_{0}\right)\right) \\
\geq_{L^{*}} \mathscr{T}^{m-n-1}\left(\mathscr{M}_{M, N}\left(g x_{n}, g x_{n+1}, \phi^{n}\left(t_{0}\right)\right),\right. \\
\mathscr{M}_{M, N}\left(g x_{n+1}, g x_{n+2}, \phi^{n+1}\left(t_{0}\right)\right), \ldots, \\
\left.\mathscr{M}_{M, N}\left(g x_{m-1}, g x_{m}, \phi^{m-1}\left(t_{0}\right)\right)\right) \\
\geq_{L^{*}} \mathscr{T}^{m-n-1}\left(\mathscr { T } _ { [ } \left(\left[\mathscr{M}_{M, N}\left(g x_{0}, g x_{1}, t_{0}\right)\right]^{2^{n-1}},\right.\right. \\
\left.\left[\mathscr{M}_{M, N}\left(g y_{0}, g y_{1}, t_{0}\right)\right]^{2^{n-1}}\right),
\end{gathered}
$$




$$
\begin{gathered}
\mathscr{T}\left(\left[\mathscr{M}_{M, N}\left(g x_{0}, g x_{1}, t_{0}\right)\right]^{2^{n}},\right. \\
\left.\left[\mathscr{M}_{M, N}\left(g y_{0}, g y_{1}, t_{0}\right)\right]^{2^{n}}\right), \ldots, \\
\mathscr{T}\left(\left[\mathscr{M}_{M, N}\left(g x_{0}, g x_{1}, t_{0}\right)\right]^{2^{m-2}},\right. \\
\left.\left.\left[\mathscr{M}_{M, N}\left(g y_{0}, g y_{1}, t_{0}\right)\right]^{2^{m-2}}\right)\right) \\
\geq_{L^{*}} \mathscr{T}\left(\left[\mathscr{M}_{M, N}\left(g x_{0}, g x_{1}, t_{0}\right)\right]^{2^{m-1}-2^{n-1}},\right. \\
\left.\left[\mathscr{M}_{M, N}\left(g y_{0}, g y_{1}, t_{0}\right)\right]^{2^{m-1}-2^{n-1}}\right) \\
\geq_{L^{*}} \mathscr{T}^{2^{m}-2^{n}-1}\left(N_{s}(\mu), \mu\right) \\
\geq_{L^{*}}\left(N_{s}(\lambda), \lambda\right),
\end{gathered}
$$

which implies that

$$
\mathscr{M}_{M, N}\left(g x_{n}, g x_{m}, t\right)>_{L^{*}}\left(N_{s}(\lambda), \lambda\right),
$$

for all $m, n \in N$ with $m>n \geq n_{0}$ and $t>0$. So $\left\{g x_{n}\right\}$ is a Cauchy sequence. Similarly, we can get that $\left\{g y_{n}\right\}$ is also a Cauchy sequence.

Step 2. Prove that $g$ and $F$ have a coupled coincidence point.

Without loss of generality, we can assume that $g(X)$ is complete; then there exist $x, y \in g(X)$ and $a, b \in X$ such that

$$
\begin{aligned}
& \lim _{n \rightarrow \infty} g x_{n}=\lim _{n \rightarrow \infty} F\left(x_{n}, y_{n}\right)=g a=x, \\
& \lim _{n \rightarrow \infty} g y_{n}=\lim _{n \rightarrow \infty} F\left(y_{n}, x_{n}\right)=g b=y .
\end{aligned}
$$

From (30), we get

$$
\begin{aligned}
& \mathscr{M}_{M, N}\left(F\left(x_{n}, y_{n}\right), F(a, b), \phi(t)\right) \\
& \quad \geq_{L^{*}} \mathscr{T}\left(\mathscr{M}_{M, N}\left(g x_{n}, g a, t\right), \mathscr{M}_{M, N}\left(g y_{n}, g b, t\right)\right) .
\end{aligned}
$$

Since $\mathscr{M}_{M, N}$ is continuous, taking limit as $n \rightarrow \infty$, we have

$$
\mathscr{M}_{M, N}(g a, F(a, b), \phi(t))=1_{L^{*}},
$$

which implies that

$$
F(a, b)=g a=x .
$$

Similarly, we can show that

$$
F(b, a)=g b=y .
$$

Since $F$ and $g$ are weakly compatible, we get that

$$
g F(a, b)=F(g a, g b), \quad g F(b, a)=F(g b, g a),
$$

which implies that

$$
g x=F(x, y), \quad g y=F(y, x) .
$$

Step 3. Prove that $g x=y$ and $g y=x$.
Since $\mathscr{T}$ is a continuous $t$-representable of $\mathrm{H}$-type. Therefore, for any $\lambda>0$, there exists a $\mu>0$ such that

$$
\mathscr{T}^{m-1}\left(N_{s}(\mu), \mu\right) \geq_{L^{*}}\left(N_{s}(\lambda), \lambda\right), \quad \forall m \in N .
$$

Since $\lim _{t \rightarrow \infty} \mathscr{M}_{M, N}(x, y, t)=1_{L^{*}}$ for all $x, y \in X$, there exists $t_{0}>0$ such that

$$
\begin{aligned}
& \mathscr{M}_{M, N}\left(g x, y, t_{0}\right) \geq_{L^{*}}\left(N_{s}(\mu), \mu\right), \\
& \mathscr{M}_{M, N}\left(g y, x, t_{0}\right) \geq_{L^{*}}\left(N_{s}(\mu), \mu\right) .
\end{aligned}
$$

On the other hand, since $\phi \in \Phi$, by condition $(\phi-3)$, we have $\sum_{n=1}^{\infty} \phi^{n}\left(t_{0}\right)<\infty$. Then, for any $t>0$, there exists $n_{0} \in N$ such that

$$
t>\sum_{k=n_{0}}^{\infty} \phi^{k}\left(t_{0}\right) .
$$

Since

$$
\begin{aligned}
\mathscr{M}_{M, N} & \left(g x, g y_{n+1}, \phi\left(t_{0}\right)\right) \\
& =\mathscr{M}_{M, N}\left(F(x, y), F\left(y_{n}, x_{n}\right), \phi\left(t_{0}\right)\right) \\
& \geq_{L^{*}} \mathscr{T}\left(\mathscr{M}_{M, N}\left(g x, g y_{n}, t_{0}\right) * \mathscr{M}_{M, N}\left(g y, g x_{n}, t_{0}\right)\right),
\end{aligned}
$$

thus

$$
\begin{aligned}
& \mathscr{M}_{M, N}\left(g x, g y_{n+1}, \phi\left(t_{0}\right)\right) \\
& \quad \geq_{L^{*}} \mathscr{T}\left(\mathscr{M}_{M, N}\left(g x, g y_{n}, t_{0}\right) * \mathscr{M}_{M, N}\left(g y, g x_{n}, t_{0}\right)\right) .
\end{aligned}
$$

Letting $n \rightarrow \infty$ in (52), by using (41), we get

$$
\begin{aligned}
& \mathscr{M}_{M, N}\left(g x, y, \phi\left(t_{0}\right)\right) \\
& \quad \geq_{L^{*}} \mathscr{T}\left(\mathscr{M}_{M, N}\left(g x, y, t_{0}\right), \mathscr{M}_{M, N}\left(g y, x, t_{0}\right)\right) .
\end{aligned}
$$

Similarly, we can get

$$
\begin{aligned}
\mathscr{M}_{M, N} & \left(g y, x, \phi\left(t_{0}\right)\right) \\
& \geq_{L^{*}} \mathscr{T}\left(\mathscr{M}_{M, N}\left(g x, y, t_{0}\right), \mathscr{M}_{M, N}\left(g y, x, t_{0}\right)\right) .
\end{aligned}
$$

From (53) and (54), we have

$$
\begin{aligned}
\mathscr{T}\left(\mathscr{M}_{M, N}\left(g x, y, \phi\left(t_{0}\right)\right), \mathscr{M}_{M, N}\left(g y, x, \phi\left(t_{0}\right)\right)\right) \\
\quad \geq_{L^{*}} \mathscr{T}\left(\left[\mathscr{M}_{M, N}\left(g x, y, t_{0}\right)\right]^{2},\left[\mathscr{M}_{M, N}\left(g y, x, t_{0}\right)\right]^{2}\right) .
\end{aligned}
$$

By this way, we can get, for all $n \in N$,

$$
\begin{aligned}
& \mathscr{T}\left(\mathscr{M}_{M, N}\left(g x, y, \phi^{n}\left(t_{0}\right)\right), \mathscr{M}_{M, N}\left(g y, x, \phi^{n}\left(t_{0}\right)\right)\right) \\
& \geq_{L^{*}} \mathscr{T}\left(\left[\mathscr{M}_{M, N}\left(g x, y, \phi^{n-1}\left(t_{0}\right)\right)\right]^{2},\right. \\
& {\left.\left[\mathscr{M}_{M, N}\left(g y, x, \phi^{n-1}\left(t_{0}\right)\right)\right]^{2}\right) } \\
& \geq_{L^{*}} \mathscr{T}\left(\left[\mathscr{M}_{M, N}\left(g x, y, t_{0}\right)\right]^{2^{n}},\left[\mathscr{M}_{M, N}\left(g y, x, t_{0}\right)\right]^{2^{n}}\right) .
\end{aligned}
$$


Thus,

$$
\begin{aligned}
\mathscr{T}\left(\mathscr{M}_{M, N}\left(g x, y, \phi^{n}\left(t_{0}\right)\right), \mathscr{M}_{M, N}\left(g y, x, \phi^{n}\left(t_{0}\right)\right)\right) \\
\quad \geq_{L^{*}} \mathscr{T}\left(\left[\mathscr{M}_{M, N}\left(g x, y, t_{0}\right)\right]^{2^{n}},\left[\mathscr{M}_{M, N}\left(g y, x, t_{0}\right)\right]^{2^{n}}\right) .
\end{aligned}
$$

Then, by (48), (49), (50), and (57), we have

$$
\begin{gathered}
\mathscr{T}\left(\mathscr{M}_{M, N}(g x, y, t), \mathscr{M}_{M, N}(g y, x, t)\right) \\
\geq_{L^{*}} \mathscr{T}\left(\mathscr{M}_{M, N}\left(g x, y, \sum_{k=n_{0}}^{\infty} \phi^{k}\left(t_{0}\right)\right)\right. \\
\left.\mathscr{M}_{M, N}\left(g y, x, \sum_{k=n_{0}}^{\infty} \phi^{k}\left(t_{0}\right)\right)\right) \\
\geq_{L^{*}} \mathscr{T}\left(\mathscr{M}_{M, N}\left(g x, y, \phi^{n_{0}}\left(t_{0}\right)\right), \mathscr{M}_{M, N}\left(g y, x, \phi^{n_{0}}\left(t_{0}\right)\right)\right) \\
\geq_{L^{*}} \mathscr{T}\left(\left[\mathscr{M}_{M, N}\left(g x, y, t_{0}\right)\right]^{2^{n_{0}}},\left[\mathscr{M}_{M, N}\left(g y, x, t_{0}\right)\right]^{2^{n_{0}}}\right) \\
\geq_{L^{*}} \mathscr{T}^{2^{n_{0}+1}-1}\left(N_{s}(\mu), \mu\right) \\
\geq_{L^{*}}\left(N_{s}(\lambda), \lambda\right) .
\end{gathered}
$$

So for any $\lambda>0$ we have

$$
\begin{array}{r}
\mathscr{T}\left(\mathscr{M}_{M, N}(g x, y, t), \mathscr{M}_{M, N}(g y, x, t)\right) \geq_{L^{*}}\left(N_{s}(\lambda), \lambda\right), \\
\forall t>0 .
\end{array}
$$

We can get that $g x=y$ and $g y=x$.

Step 4. Prove that $x=y$.

Since $\mathscr{T}$ is a continuous $t$-representable of H-type. Therefore, for any $\lambda>0$, there exists a $\mu>0$ such that

$$
\mathscr{T}^{m-1}\left(N_{s}(\mu), \mu\right) \geq_{L^{*}}\left(N_{s}(\lambda), \lambda\right), \quad \forall m \in N .
$$

Since $\lim _{t \rightarrow \infty} \mathscr{M}_{M, N}(x, y, t)=1_{L^{*}}$ for all $x, y \in X$, there exists $t_{0}>0$ such that

$$
\mathscr{M}_{M, N}\left(x, y, t_{0}\right) \geq_{L^{*}}\left(N_{s}(\mu), \mu\right) .
$$

On the other hand, since $\phi \in \Phi$, by condition $(\phi-3)$, we have $\sum_{n=1}^{\infty} \phi^{n}\left(t_{0}\right)<\infty$. Then, for any $t>0$, there exists $n_{0} \in N$ such that

$$
t>\sum_{k=n_{0}}^{\infty} \phi^{k}\left(t_{0}\right)
$$

Since, for $t_{0}>0$,

$$
\begin{aligned}
& \mathscr{M}_{M, N}\left(g x_{n+1}, g y_{n+1}, \phi\left(t_{0}\right)\right) \\
& \quad=\mathscr{M}_{M, N}\left(F\left(x_{n}, y_{n}\right), F\left(y_{n}, x_{n}\right), \phi\left(t_{0}\right)\right) \\
& \geq_{L^{*}} \mathscr{T}\left(\mathscr{M}_{M, N}\left(g x_{n}, g y_{n}, t_{0}\right), \mathscr{M}_{M, N}\left(g y_{n}, g x_{n}, t_{0}\right)\right),
\end{aligned}
$$

thus

$$
\begin{array}{r}
\mathscr{M}_{M, N}\left(g x_{n+1}, g y_{n+1}, \phi\left(t_{0}\right)\right) \\
\geq_{L^{*}} \mathscr{T}\left(\mathscr{M}_{M, N}\left(g x_{n}, g y_{n}, t_{0}\right),\right. \\
\left.\mathscr{M}_{M, N}\left(g y_{n}, g x_{n}, t_{0}\right)\right) .
\end{array}
$$

Letting $n \rightarrow \infty$ in the above inequality, we get

$$
\begin{aligned}
& \mathscr{M}_{M, N}\left(x, y, \phi\left(t_{0}\right)\right) \\
& \quad \geq_{L^{*}} \mathscr{T}\left(\mathscr{M}_{M, N}\left(x, y, t_{0}\right), \mathscr{M}_{M, N}\left(y, x, t_{0}\right)\right) .
\end{aligned}
$$

Thus, by (60), (61), (62), and (65), we have

$$
\begin{aligned}
\mathscr{M}_{M, N}(x, y, t) \\
\quad \geq_{L^{*}} \mathscr{M}_{M, N}\left(x, y, \sum_{k=n_{0}}^{\infty} \phi^{k}\left(t_{0}\right)\right) \\
\quad \geq_{L^{*}} \mathscr{M}_{M, N}\left(x, y, \phi^{n_{0}}\left(t_{0}\right)\right) \\
\quad \geq_{L^{*}} \mathscr{T}\left(\left[\mathscr{M}_{M, N}\left(x, y, t_{0}\right)\right]^{2^{n_{0}}-1},\left[\mathscr{M}_{M, N}\left(y, x, t_{0}\right)\right]^{2^{n_{0}}-1}\right) \\
\quad \geq_{L^{*}} \mathscr{T}^{2^{n_{0}+1}-3}\left(N_{s}(\mu), \mu\right) \\
\geq_{L^{*}}\left(N_{s}(\lambda), \lambda\right),
\end{aligned}
$$

which implies that $x=y$. Thus, we have proved that $F$ and $g$ have a unique common fixed point in $X$. This completes the proof of Theorem 31.

Taking $g=I$ (the identity mapping) in Theorem 31, we get the following consequence.

Corollary 32. Let $\left(X, \mathscr{M}_{M, N}, \mathscr{T}\right)$ be a complete modified intuitionistic fuzzy metric space, where $\mathscr{T}$ is continuous $t$ representable of $H$-type satisfying (22). Let $F: X \times X \rightarrow X$ and there exist $\phi \in \Phi$ such that

$$
\begin{array}{rl}
\mathscr{M}_{M, N} & (F(x, y), F(u, v), \phi(t)) \\
\geq_{L^{*}} & \mathscr{T}\left(\mathscr{M}_{M, N}(x, u, t), \mathscr{M}_{M, N}(y, v, t)\right),
\end{array}
$$

for all $x, y, u, v \in X$ and $t>0$. Then there exists $x \in X$ such that $x=F(x, x)$.

Put $\phi(t)=k t$, where $0<k<1$, in Theorem 31; we get the following.

Corollary 33. Let $\left(X, \mathscr{M}_{M, N}, \mathscr{T}\right)$ be a modified intuitionistic fuzzy metric space such that $\mathscr{M}_{M, N}$ has p-property. Let $F: X \times$ $X \rightarrow X$ and $g: X \rightarrow X$ be two functions such that

$$
\begin{array}{rl}
\mathscr{M}_{M, N} & (F(x, y), F(u, v), k t) \\
\geq_{L^{*}} & \mathscr{T}\left(\mathscr{M}_{M, N}(g x, g u, t), \mathscr{M}_{M, N}(g y, g v, t)\right),
\end{array}
$$

for all $x, y, u, v \in X$ and $t>0$, where $0<k<1$. Suppose that $F(X \times X) \subseteq g(X)$ and $F(X \times X)$ or $g(X)$ is complete. Then there exists a unique $x \in X$ such that $x=g(x)=F(x, x)$. 
Remark 34. Comparing Theorem 31 in the present paper with Theorem 3.1 in [13], we can see that Theorem 31 is a genuine generalization of Theorem 3.1 in the sense that

(1) we only use the completeness of $g(X)$ or $F(X \times X)$;

(2) we drop off the continuity of $g$;

(3) the concept of compatible mappings has been replaced by weakly compatible mappings.

Next, we give an example to demonstrate Theorem 31.

Example 35. Let $X=\{0,1,1 / 2,1 / 3, \ldots, 1 / n, \ldots\}$ and $\mathscr{T}(a, b)=\left(\min \left\{a_{1}, b_{1}\right\}, \max \left\{a_{2}, b_{2}\right\}\right)$, for all $a=\left(a_{1}, a_{2}\right)$ and $b=\left(b_{1}, b_{2}\right) \in L^{*}$. Define

$$
\begin{array}{r}
\mathscr{M}_{M, N}(x, y, t)=\left(\frac{t}{t+|x-y|}, \frac{|x-y|}{t+|x-y|}\right), \\
\forall x, y \in X, t>0 .
\end{array}
$$

Then $\left(X, \mathscr{M}_{M, N}, \mathscr{T}\right)$ is a modified intuitionistic fuzzy metric space. Let $\phi(t)=t / 2$. Let $g: X \rightarrow X$ and $F: X \times X \rightarrow X$ be defined as

$$
\begin{gathered}
g(x)= \begin{cases}0, & x=0, \\
1, & x=\frac{1}{2 n+1}, \\
\frac{1}{2 n+1}, & x=\frac{1}{2 n},\end{cases} \\
F(x, y)= \begin{cases}\frac{1}{(2 n+1)^{4}}, & (x, y)=\left(\frac{1}{2 n}, \frac{1}{2 n}\right), \\
0, & \text { otherwise. }\end{cases}
\end{gathered}
$$

Let $x_{n}=y_{n}=1 / 2 n$. We have

$$
\begin{gathered}
g x_{n}=\frac{1}{2 n+1} \longrightarrow 0, \\
F\left(x_{n}, y_{n}\right)=\frac{1}{(2 n+1)^{4}} \longrightarrow 0 \text { as } n \longrightarrow \infty,
\end{gathered}
$$

but

$$
\begin{gathered}
\mathscr{M}_{M, N}\left(F\left(g x_{n}, g y_{n}\right), g F\left(x_{n}, y_{n}\right), t\right) \\
=\mathscr{M}_{M, N}(0,1, t) \nrightarrow 1_{L^{*}},
\end{gathered}
$$

so $g$ and $F$ are not compatible. From $F(x, y)=g(x)$ and $F(y, x)=g(y)$, we can get $(x, y)=(0,0)$ and we have $g F(0,0)=F(g 0, g 0)$, which implies that $F$ and $g$ are weakly compatible. The following result is easy to be verified:

$$
\begin{aligned}
& \frac{t}{X+t} \geq \min \left\{\frac{t}{Y+t}, \frac{t}{Z+t}\right\}, \\
& \frac{X}{X+t} \leq \max \left\{\frac{Y}{Y+t}, \frac{Z}{Z+t}\right\}, \\
& \Longleftrightarrow X \leq \max \{Y, Z\}, \quad \forall X, Y, Z \geq 0, t>0 .
\end{aligned}
$$

By the definition of $\mathscr{M}_{M, N}, \phi$ and the result above, we can get inequality (30):

$$
\begin{aligned}
\mathscr{M}_{M, N} & (F(x, y), F(u, v), \phi(t)) \\
& \geq_{L^{*}} \mathscr{T}\left(\mathscr{M}_{M, N}(g x, g u, t), \mathscr{M}_{M, N}(g y, g v, t)\right),
\end{aligned}
$$

which is equivalent to the following:

$$
2|F(x, y)-F(u, v)| \leq \max \{|g x-g u|,|g y-g v|\} .
$$

Now, we verify inequality (75). Let $A=\{1 / 2 n, n \in N\}, B=$ $X-A$. By the symmetry and without loss of generality, $(x, y),(u, v)$ have six possibilities.

Case 1. $(x, y) \in B \times B,(u, v) \in B \times B$. It is obvious that (75) holds.

Case 2. $(x, y) \in B \times B,(u, v) \in B \times A$. It is obvious that (75) holds.

Case 3. $(x, y) \in B \times B,(u, v) \in A \times A$. If $u \neq v$, (75) holds. If $u=v$, let $u=v=1 / 2 n$, and then

$$
\begin{gathered}
2|F(x, y)-F(u, v)|=\frac{2}{(2 n+1)^{4}}, \\
\max \{|g x-g u|,|g y-g v|\}=\frac{2 n}{2 n+1},
\end{gathered}
$$

which implies that (75) holds.

Case 4. $(x, y) \in B \times A,(u, v) \in B \times A$. It is obvious that (75) holds.

Case 5. $(x, y) \in B \times A,(u, v) \in A \times A$. If $u \neq v$, (75) holds. If $u=v$, let $x \in B, y=1 / 2 j, u=v=1 / 2 n$, and then

$$
2|F(x, y)-F(u, v)|=\frac{2}{(2 n+1)^{4}},
$$

$$
\begin{aligned}
\max & \{|g x-g u|,|g y-g v|\} \\
& =\max \left\{\frac{1}{2 n+1},\left|\frac{1}{2 j+1}-\frac{1}{2 n+1}\right|\right\},
\end{aligned}
$$

or

$$
\begin{aligned}
\max & \{|g x-g u|,|g y-g v|\} \\
& =\max \left\{\frac{2 n}{2 n+1},\left|\frac{1}{2 j+1}-\frac{1}{2 n+1}\right|\right\} .
\end{aligned}
$$

So, (75) holds.

Case 6. $(x, y) \in A \times A$ and $(u, v) \in A \times A$. If $x \neq y$ and $u \neq v$, (75) holds. If $x \neq y, u=v$, let $x=1 / 2 i, y=1 / 2 j, i \neq j$, and $u=v=1 / 2 n$. Then

$$
\begin{gathered}
2|F(x, y)-F(u, v)|=\frac{2}{(2 n+1)^{4}}, \\
\max \{|g x-g u|,|g y-g v|\} \\
=\max \left\{\left|\frac{1}{2 i+1}-\frac{1}{2 n+1}\right|,\left|\frac{1}{2 j+1}-\frac{1}{2 n+1}\right|\right\} .
\end{gathered}
$$


So, (75) holds. If $x=y$ and $u=v$, let $x=y=1 / 2 i, u=v=$ $1 / 2 n$. Then

$$
\begin{gathered}
2|F(x, y)-F(u, v)|=2\left|\frac{1}{(2 i+1)^{4}}-\frac{1}{(2 n+1)^{4}}\right| \\
\max \{|g x-g u|,|g y-g v|\} \\
=\frac{1}{2 i+1}-\frac{1}{2 n+1} .
\end{gathered}
$$

So, (75) holds. Then all the conditions in Theorem 31 are satisfied and 0 is the unique common fixed point of $g$ and F.

\section{Application to Integral Equations}

As an application of the coupled fixed point theorems established in Section 3 of our paper, we study the existence and uniqueness of the solution to a Fredholm nonlinear integral equation. We will consider the following integral equation:

$$
\begin{aligned}
x(p)= & \int_{a}^{b}\left(K_{1}(p, q)+K_{2}(p, q)\right) \\
& \times[f(q, x(q))+g(q, x(q))] d q+h(p),
\end{aligned}
$$

for all $p \in I=[a, b]$.

Let $\Theta$ denote the set of all functions $\theta:[0,+\infty) \rightarrow$ $[0,+\infty)$ satisfying the following:

$\left(\mathrm{i}_{\theta}\right) \theta$ is nondecreasing;

$\left(\mathrm{ii}_{\theta}\right) \theta(p) \leq p$.

We assume that the functions $K_{1}, K_{2}, f, g$ fulfill the following conditions.

Assumption 36. (i) Consider

$$
K_{1}(p, q) \geq 0, \quad K_{2}(p, q) \leq 0, \quad \forall p, q \in I .
$$

(ii) There exists positive numbers $\lambda, \mu$, and $\theta \in \Theta$ such that, for all $x, y \in R$ with $x \geq y$, the following conditions hold:

$$
\begin{aligned}
& 0 \leq f(q, x)-f(q, y) \leq \lambda \theta(x-y), \\
& -\mu \theta(x-y) \leq g(q, x)-g(q, y) \leq 0 .
\end{aligned}
$$

(iii) Consider

$$
\max \{\lambda, \mu\} \sup _{p \in I} \int_{a}^{b}\left[K_{1}(p, q)-K_{2}(p, q)\right] d q \leq \frac{1}{8}
$$

Theorem 37. Consider the integral equation (81) with $K_{1}, K_{2} \in C(I \times I, \mathbb{R}), f, g \in C(I \times \mathbb{R}, \mathbb{R})$, and $h \in C(I, R)$. Suppose that Assumption 36 is satisfied. Then the integral equation (81) has a unique solution in $C(I, R)$.

Proof. Consider $X=C(I, R)$. It is easy to check that $\left(X, \mathscr{M}_{M, N}, \mathscr{T}\right)$ is a complete modified intuitionistic fuzzy metric space with respect to the modified intuitionistic fuzzy metric:

$$
\begin{array}{r}
\mathscr{M}_{M, N}(x, y, t)=\left(\frac{t}{t+|x-y|}, \frac{|x-y|}{t+|x-y|}\right), \\
\forall x, y \in X, t>0,
\end{array}
$$

for all $x, y \in X$ and $t>0$ with $\mathscr{T}(a, b)=$ $\left(\min \left\{a_{1}, b_{1}\right\}, \max \left\{a_{2}, b_{2}\right\}\right)$, for all $a=\left(a_{1}, a_{2}\right)$ and $b=\left(b_{1}, b_{2}\right) \in$ $L^{*}$. Define now the mapping $F: X \times X \rightarrow X$ by

$$
\begin{array}{r}
F(x, y)(p)=\int_{a}^{b} K_{1}(p, q)[f(q, x(q))+g(q, y(q))] d q \\
+\int_{a}^{b} K_{2}(p, q)[f(q, y(q)) \\
+g(q, x(q))] d q+h(p),
\end{array}
$$

for all $p \in I$ and $\phi(t)=t / 2$ for all $t>0$. Now, for all $x, y, u, v \in$ $X$, using (83), we have

$$
\begin{aligned}
& F(x, y)(p)-F(u, v)(p) \\
& =\int_{a}^{b} K_{1}(p, q)[f(q, x(q))+g(q, y(q))] d q \\
& +\int_{a}^{b} K_{2}(p, q)[f(q, y(q))+g(q, x(q))] d q \\
& -\int_{a}^{b} K_{1}(p, q)[f(q, u(q))+g(q, v(q))] d q \\
& -\int_{a}^{b} K_{2}(p, q)[f(q, v(q))+g(q, u(q))] d q \\
& =\int_{a}^{b} K_{1}(p, q)[f(q, x(q))-f(q, u(q)) \\
& +g(q, y(q))-g(q, v(q))] d q \\
& +\int_{a}^{b} K_{2}(p, q)[f(q, y(q))-f(q, v(q)) \\
& +g(q, x(q))-g(q, u(q))] d q \\
& =\int_{a}^{b} K_{1}(p, q)[(f(q, x(q))-f(q, u(q))) \\
& -(g(q, v(q))-g(q, y(q)))] d q \\
& -\int_{a}^{b} K_{2}(p, q)[(f(q, v(q))-f(q, y(q))) \\
& -(g(q, x(q))-g(q, u(q)))] d q \\
& \leq \int_{a}^{b} K_{1}(p, q)[\lambda \theta(x(q)-u(q)) \\
& +\mu \theta(v(q)-y(q))] d q
\end{aligned}
$$




$$
\begin{aligned}
-\int_{a}^{b} K_{2}(p, q)[ & \lambda \theta(v(q)-y(q)) \\
& +\mu \theta(x(q)-u(q))] d q .
\end{aligned}
$$

Thus

$$
\begin{aligned}
& F(x, y)(p)-F(u, v)(p) \\
& \leq \int_{a}^{b} K_{1}(p, q)[\lambda \theta(x(q)-u(q))+\mu \theta(v(q)-y(q))] d q \\
&-\int_{a}^{b} K_{2}(p, q)[\lambda \theta(v(q)-y(q)) \\
&+\mu \theta(x(q)-u(q))] d q .
\end{aligned}
$$

Since the function $\theta$ is nondecreasing, so we have

$$
\begin{aligned}
& \theta(x(q)-u(q)) \leq \theta(|x(q)-u(q)|) \\
& \theta(v(q)-y(q)) \leq \theta(|v(q)-y(q)|) .
\end{aligned}
$$

Hence, by (88), in view of the fact that $K_{2}(p, q) \leq 0$, we get

$$
\begin{aligned}
& |F(x, y)(p)-F(u, v)(p)| \\
& \leq \int_{a}^{b} K_{1}(p, q)[\lambda \theta(|x(q)-u(q)|) \\
& +\mu \theta(|v(q)-y(q)|)] d q \\
& \quad-\int_{a}^{b} K_{2}(p, q)[\lambda \theta(|v(q)-y(q)|) \\
& +\mu \theta(|x(q)-u(q)|)] d q \\
& \leq \int_{a}^{b} K_{1}(p, q)[\max \{\lambda, \mu\} \theta(|x(q)-u(q)|) \\
& \quad+\max \{\lambda, \mu\} \theta(|v(q)-y(q)|)] d q \\
& \quad-\int_{a}^{b} K_{2}(p, q)[\max \{\lambda, \mu\} \theta(|v(q)-y(q)|) \\
& \quad+\max \{\lambda, \mu\} \theta(|x(q)-u(q)|)] d q,
\end{aligned}
$$

as all the quantities on the right hand side of (88) are nonnegative. Now, by using (84), we get

$$
\begin{aligned}
& |F(x, y)-F(u, v)| \\
& \leq \max \{\lambda, \mu\} \int_{a}^{b}\left[K_{1}(p, q)-K_{2}(p, q)\right] d q \\
& \cdot[\theta(|x(q)-u(q)|)+\theta(|v(q)-y(q)|)]
\end{aligned}
$$

$$
\begin{aligned}
& \leq \max \{\lambda, \mu\} \sup _{p \in I} \int_{a}^{b}\left[K_{1}(p, q)-K_{2}(p, q)\right] d q \\
& \cdot[\theta(|x(q)-u(q)|)+\theta(|v(q)-y(q)|)] \\
& \leq \frac{\theta(|x-u|)+\theta(|v-y|)}{8} .
\end{aligned}
$$

Thus

$$
2|F(x, y)-F(u, v)| \leq \frac{\theta(|x-u|)+\theta(|v-y|)}{4} .
$$

Now, since $\theta$ is nondecreasing, we have

$$
\begin{aligned}
& \theta(|x-u|) \leq \theta(|x-u|+|y-v|), \\
& \theta(|y-v|) \leq \theta(|x-u|+|y-v|),
\end{aligned}
$$

which, by $\left(\mathrm{ii}_{\theta}\right)$, implies

$$
\begin{aligned}
\frac{\theta(|x-u|)+\theta(|y-v|)}{2} & \leq \theta(|x-u|+|y-v|) \\
& \leq|x-u|+|y-v| \\
& \leq 2 \max \{|x-u|,|y-v|\},
\end{aligned}
$$

and so

$$
\frac{\theta(|x-u|)+\theta(|y-v|)}{4} \leq \max \{|x-u|,|y-v|\} .
$$

Thus, by (92) and (95), we get

$$
2|F(x, y)-F(u, v)| \leq \max \{|x-u|,|y-v|\} .
$$

Now, by (96) and (73), it follows that

$$
\begin{gathered}
\mathscr{M}_{M, N}(F(x, y), F(u, v), \phi(t)) \\
=\mathscr{M}_{M, N}\left(F(x, y), F(u, v), \frac{t}{2}\right) \\
=\left(\frac{t / 2}{t / 2+|F(x, y)-F(u, v)|},\right. \\
=\left(\frac{|F(x, y)-F(u, v)|}{t / 2+|F(x, y)-F(u, v)|}\right) \\
\frac{t+2|F(x, y)-F(u, v)|}{t|F(x, y)-F(u, v)|} \\
\geq_{L^{*}}\left(\frac{t|F(x, y)-F(u, v)|}{t+2 \mid F(\max \{|x-u|,|y-v|\}},\right. \\
\left.\frac{\max \{|x-u|,|y-v|\}}{t+\max \{|x-u|,|y-v|\}}\right)
\end{gathered}
$$




$$
\begin{aligned}
& \geq_{L^{*}}\left(\min \left\{\frac{t}{t+|x-u|}, \frac{t}{t+|y-v|}\right\},\right. \\
& \left.\quad \max \left\{\frac{|x-u|}{t+|x-u|}, \frac{|y-v|}{t+|y-v|}\right\}\right) \\
& \geq_{L^{*}} \mathscr{T}\left(\mathscr{M}_{M, N}(x, u, t), \mathscr{M}_{M, N}(y, v, t)\right),
\end{aligned}
$$

which is the condition in (67), shows that all hypotheses of Corollary 32 are satisfied. This proves that $F$ has a unique fixed point $\tilde{x} \in X$; that is, $\tilde{x}=F(\tilde{x}, \tilde{x})$, and therefore $\tilde{x} \in C(I, R)$ is the unique solution of the integral equation (81).

\section{Conflict of Interests}

The authors declare that there is no conflict of interests regarding the publication of this paper.

\section{References}

[1] I. Kramosil and J. Michalek, "Fuzzy metric and statistical metric spaces,” Kybernetika, vol. 11, no. 5, pp. 336-344, 1975.

[2] A. George and P. Veeramani, "On some results in fuzzy metric spaces,” Fuzzy Sets and Systems, vol. 64, no. 3, pp. 395-399, 1994.

[3] K. T. Atanassov, "Intuitionistic fuzzy sets," Fuzzy Sets and Systems, vol. 20, no. 1, pp. 87-96, 1986.

[4] C. Alaca, D. Turkoglu, and C. Yildiz, "Fixed points in intuitionistic fuzzy metric spaces," Chaos, Solitons \& Fractals, vol. 29, no. 5, pp. 1073-1078, 2006.

[5] J. H. Park, "Intuitionistic fuzzy metric spaces," Chaos, Solitons \& Fractals, vol. 22, no. 5, pp. 1039-1046, 2004.

[6] V. Gregori, S. Romaguera, and P. Veeramani, "A note on intuitionistic fuzzy metric spaces," Chaos, Solitons \& Fractals, vol. 28, no. 4, pp. 902-905, 2006.

[7] R. Saadati, S. Sedghi, and N. Shobe, "Modified intuitionistic fuzzy metric spaces and some fixed point theorems," Chaos, Solitons \& Fractals, vol. 38, no. 1, pp. 36-47, 2008.

[8] T. G. Bhaskar and V. Lakshmikantham, "Fixed point theorems in partially ordered metric spaces and applications," Nonlinear Analysis: Theory, Methods and Applications, vol. 65, no. 7, pp. 1379-1393, 2006.

[9] V. Lakshmikantham and L. Ciric, "Coupled fixed point theorems for nonlinear contractions in partially ordered metric spaces," Nonlinear Analysis: Theory, Methods and Applications, vol. 70, no. 12, pp. 4341-4349, 2009.

[10] S. Sedghi, I. Altun, and N. Shobe, "Coupled fixed point theorems for contractions in fuzzy metric spaces," Nonlinear Analysis: Theory, Methods and Applications, vol. 72, no. 3-4, pp. 12981304, 2010.

[11] X. Q. Hu, "Common coupled fixed point theorems for contractive mappings in fuzzy metric spaces," Fixed Point Theory and Applications, vol. 2011, Article ID 363716, 14 pages, 2011.

[12] X. Q. Hu, M. X. Zheng, B. Damjanovic, and X. F. Shao, "Common coupled fixed point theorems for weakly compatible mappings in fuzzy metric spaces," Fixed Point Theory and Applications, vol. 2013 article 220, 2013.

[13] B. Deshpande, S. Sharma, and A. Handa, "Common coupled fixed point theorems for nonlinear contractive condition on intuitionistic fuzzy metric space with application to integral equations," Journal of the Korean Society of Mathematical Education B: The Pure and Applied Mathematics, vol. 20, no. 3, pp. 159-180, 2013.

[14] G. Deschrijver and E. E. Kerre, "On the relationship between some extensions of fuzzy set theory," Fuzzy Sets and Systems, vol. 133, no. 2, pp. 227-235, 2003.

[15] G. Deschrijver, C. Cornelis, and E. E. Kerre, "On the representation of intuitionistic fuzzy t-norms and t-conorms," IEEE Transactions on Fuzzy Systems, vol. 12, no. 1, pp. 45-61, 2004.

[16] J. X. Fang, "On fixed point theorems in fuzzy metric spaces," Fuzzy Sets and Systems, vol. 46, no. 1, pp. 107-113, 1992.

[17] R. Saadati and J. H. Park, "On the modified intuitionistic fuzzy topological spaces," Chaos, Solitons \& Fractals, vol. 27, no. 2, pp. 331-334, 2006.

[18] J. X. Fang, "Common fixed point theorems of compatible and weakly compatible maps in Menger spaces," Nonlinear Analysis: Theory, Methods and Applications, vol. 71, no. 5-6, pp. 1833-1843, 2009.

[19] M. Abbas, M. A. Khan, and S. Radenovic, "Common coupled fixed point theorems in cone metric spaces for w-compatible mappings," Applied Mathematics and Computation, vol. 217, no. 1, pp. 195-202, 2010. 

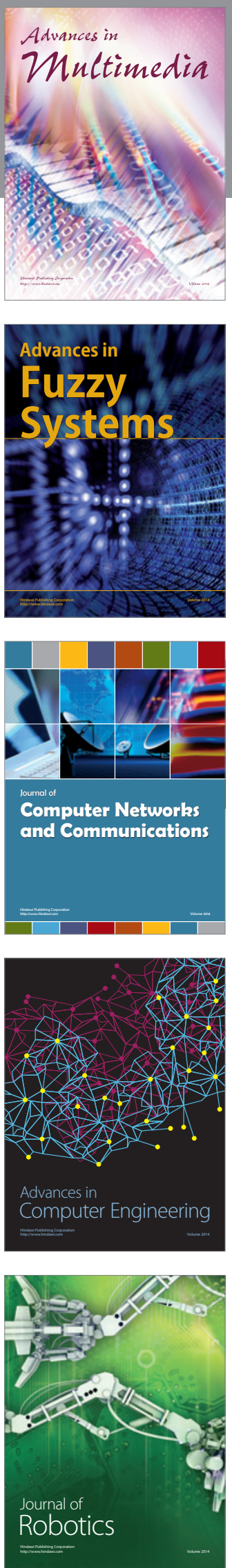

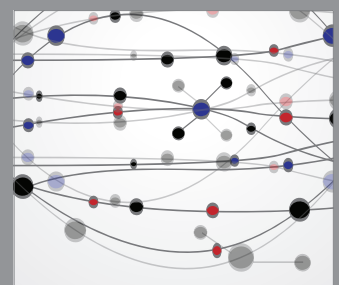

The Scientific World Journal
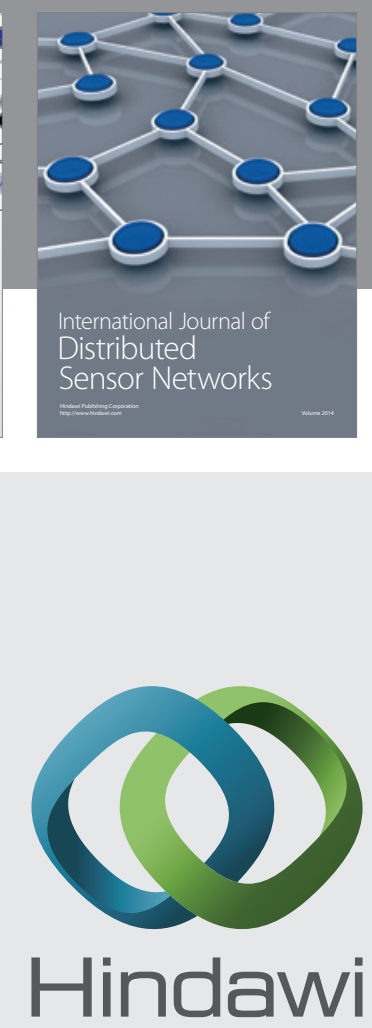

Submit your manuscripts at

http://www.hindawi.com
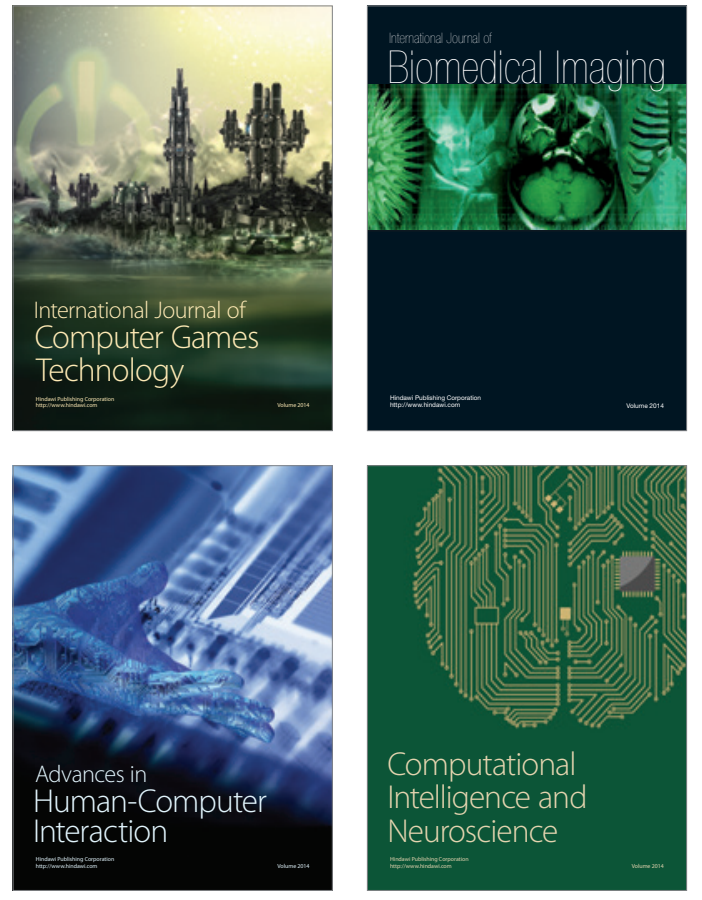
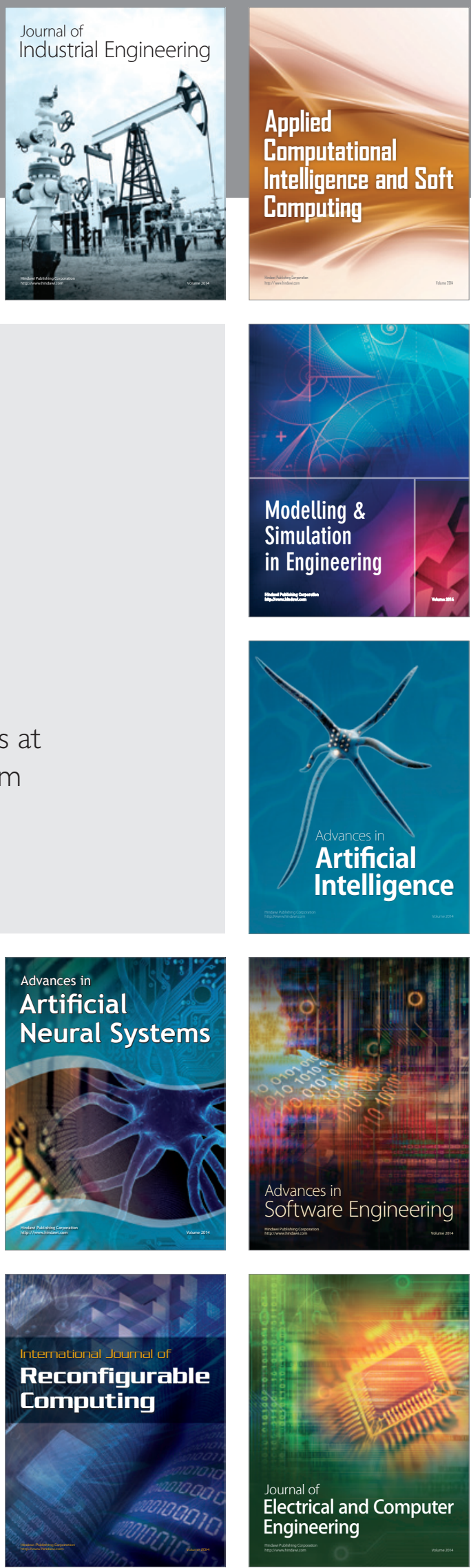